\title{
FLIPPED CLASSROOM Y DERECHO FINANCIERO: UN BINOMIO NECESARIO ${ }^{1}$
}

\author{
MARÍA DEL MAR SOTO MOYA \\ Dra. Derecho Financiero y Tributario \\ marsotomoya@uma.es \\ Universidad de Málaga
}

\begin{abstract}
Resumen:
En el contexto universitario actual, en el que el alumnado realiza un uso constante de las TICs, se hace imprescindible la utilización por parte del docente de los escenarios virtuales de aprendizaje. Estos instrumentos resultan idóneos en la enseñanza del Derecho Financiero, en el que la indisolubilidad entre contenidos teóricos y prácticos provoca la necesidad de conocer la realidad existente. Este objetivo puede lograrse a través de la metodología de la clase invertida, que convierte al alumnado en un sujeto activo del aprendizaje, superando la consideración de simple oyente en las clases presenciales.
\end{abstract}

Palabras claves: Clase invertida, Derecho Financiero, innovación, metodología, escenario virtual.

\begin{abstract}
:
In the current university context, in which students make constant use of ICT, it is essential to use the virtual learning environments. These instruments are ideal in the teaching of Financial Law, in which the indissolubility between theoretical and practical contents provokes the need to know the existing reality. This objective can be achieved through the methodology of the flipped classroom, which turns the student into an active subject of learning, surpassing the consideration of simple listener in face-to-face classes.
\end{abstract}

Keywords: Flipped classroom, Financial Law, innovation, methodology, virtual environment.

Sumario: 1. Introducción- 2. Concepto y características de la clase invertida3. Herramientas para la realización de la clase invertida- 4. La conveniente utilización de la clase invertida en las asignaturas de Derecho Financiero y Tributario- 5. A modo de reflexión. Bibliografía.

\footnotetext{
1 Este artículo se enmarca en el Proyecto de Innovación Educativa PIE 17-028 "Los medios de información como recurso didáctico en la enseñanza del Derecho", concedido por la Universidad de Málaga en la convocatoria 2017-2019.

REJIE Nueva época: Revista Jurídica de Investigación e Innovación Educativa Núm.18, Junio 2018, pp. 31-42 


\section{Introducción.}

La Universidad española ha sufrido en la últimas décadas importantes cambios en cuanto a la ordenación de las enseñanzas, y por tanto, también respecto a la metodología docente ${ }^{2}$.

Con la Declaración de Bolonia de 19 de junio de 1999, se instauró un sistema educativo común con el doble objetivo de lograr una mayor compatibilidad y cotejo de los sistemas nacionales de educación superior, por una parte, y de incrementar la competitividad del sistema de enseñanza superior europeo por otra, potenciando su capacidad de atracción hacia el exterior.

Para ello, se estableció un sistema de titulaciones comparables, un sistema común de créditos (ECTS) o la mayor promoción de la movilidad, entre otras herramientas. En la actualidad, son evidentes las implicaciones que para el ejercicio de la docencia se han derivado de la Declaración de Bolonia, de la implantación del crédito europeo y, en general, del proceso de adaptación al Espacio Europeo de Enseñanza Superior (EEES) ${ }^{3}$. Pese a que, en un primer momento, uno de los propósitos de los nuevos Planes de Estudios fue la limitación del número de estudiantes por grupo, con el objetivo de facilitar la realización de trabajos prácticos y superar la clase magistral como metodología principal, lo cierto es que en la actualidad, al menos en titulaciones como el Grado en Derecho, en muchas asignaturas se supera sin dificultad la cifra de cien alumnos/as.

Esta circunstancia, provocada entre otras razones por la crisis económica por la que hemos atravesado, dificulta, en mi opinión, la realización de prácticas en clase, así como la adquisición de determinadas competencias por parte de los discentes. Por ejemplo, dentro de las competencias básicas del Grado en Derecho de la Universidad de Málaga, en el que imparto diferentes asignaturas, una de las competencias básicas es "que los estudiantes puedan transmitir información, ideas, problemas y soluciones a un público tanto especializado como no especializado" o, dentro de las competencias transversales: trabajo en equipo, liderazgo, aprendizaje autónomo... Resulta complicado, en grupos de más de cien alumnos/as y en el ámbito espacial y temporal de la clase presencial, realizar prácticas o dinámicas de grupo que permitan al alumno/a adquirir ese tipo de competencias, además de los conocimientos básicos sobre el temario establecido.

Es en este punto en el que, como docentes, debemos preguntarnos: ¿Es posible establecer una nueva metodología que permita un aprendizaje más práctico por parte del alumnado? ¿Es posible la adaptación de la enseñanza a las nuevas realidades? La respuesta debe ser positiva, existen instrumentos que posibilitan, dentro de los parámetros de los Planes de Estudios establecidos, un aprendizaje activo, autónomo y reflexivo del alumno/a. Y entre estas herramientas se encuentra el modelo de la clase invertida o flipped classroom.

\footnotetext{
${ }^{2}$ Una recopilación de toda la normativa aplicable en el ámbito universitario puede consultarse en ALCUBILlA, E.A. (2016): Código de Universidades. Códigos electrónicos. Agencia Estatal Boletín Oficial del Estado.

${ }^{3}$ En nuestro país, el Gobierno aprobó el RD 1393/2007, de 29 de octubre, por el que se establece la ordenación de las enseñanzas universitarias oficiales, seguido de otras disposiciones complementarias, con el que se declararon derogados los RD 55 y 56 de 2005.
} 


\section{Concepto y características de la clase invertida.}

La necesidad de renovación metodológica en el ámbito universitario es una realidad incuestionable hoy en día. Son numerosos los estudios que evidencian la obsolescencia de la clase magistral tradicional, en la que la atención de los alumnos disminuye después de los primeros quince minutos de clase ${ }^{4}$. Al respecto, establece IMBERNÓN que "si bien, es cierto, que no se debe dejar de lado el tradicional objetivo de transmitir conocimientos específicos, aparecen objetivos nuevos y se modifica la relación de prioridades. Ante estos cambios es necesario adoptar metodologías didácticas eficaces para afrontar los nuevos requerimientos".

Entre estos requerimientos destaca la necesidad de que el alumno, y más en el contexto universitario, aprenda no solo desde una perspectiva teórica o conceptual, sino desde un enfoque práctico y aplicable tanto a su ámbito profesional como en la propia sociedad civil. Para que este objetivo pueda cumplirse, es necesario que el trabajo del alumno se traslade fuera del aula pues, como señalaba anteriormente, las insuficientes horas de clase presencial, unido al elevado número de alumnos, provoca enormes dificultades para acometer esta tarea.

Sin embargo, estos obstáculos pueden ser franqueables a través de la metodología de la clase invertida, que facilita las condiciones necesarias para que los alumnos aprendan por sí mismos. El alumnado se convierte en el centro de la clase, adquiriendo un rol mucho más activo, superando el papel de simple oyente y participando en las actividades presenciales. Por su parte, el profesor/a supera también el rol de "busto parlante" con preeminencia de métodos expositivos para convertirse en un moderador/a de la actividad, aportando sugerencias y recursos para la resolución de los problemas ${ }^{6}$.

Uno de los primeros ejemplos de flipped classroom o clase invertida fue el método utilizado por Bergmann y Sams, profesores del Woodland Park High School que, ante el absentismo y la apatía que mostraba el alumnado, pensaron que una buena solución sería grabar sus clases y colgarlas en YouTube como material de apoyo para que pudieran visualizarse en cualquier momento. Después utilizaban las clases para la realización de actividades prácticas y no para una explicación tradicional. En este sentido, como señalan SÁNCHEZ VERA, SOLANI FERNÁNDEZ y GONZÁLEZ CALATAYUD "Flipped Classroom ha permitido que los alumnos identifiquen el aprendizaje como objetivo último de la actividad, y no la realización de la tarea, o lo que es peor, la superación del examen",7.

\footnotetext{
${ }^{4}$ Sobre este particular, BUNCE, D. M., FLENS, E. A., y NEILES, K. Y. (2010): "How long can students pay attention in class? A study of student attention decline using clickers". Journal of Chemical Education, 87(12), págs. 1438-1443.

${ }^{5}$ IMBERNÓN, F. (2018): "Cómo mejorar las sesiones expositivas", Campus virtual UMA.

${ }^{6}$ En este sentido, IMBERNÓN, F. (2009): "Mejorar la enseñanza y el aprendizaje en la Universidad", Cuadernos de Docencia Universitaria, $\mathrm{N}^{\circ} 14$.

${ }^{7}$ SÁNCHEZ VERA, M.M.; SOLANO FERNÁNDEZ, I.M. y GONZÁLEZ CALATAYUD, V. (2016): "FLIPPEDTIC: Una experiencia de Flipped Classroom con alumnos de Magisterio", Revista Latinoamericana de Tecnología Educativa, Vol 15 (3), pág. 71.
} 
La clase invertida supone la incorporación de información por el alumno de forma previa a la clase presencial, de manera que en esta se pueden realizar actividades prácticas y de aplicación a la vida real. Como indican SÁNCHEZ RODRÍGUEZ, RUIZ-PALMERO y SÁNCHEZ-VEGA “es un enfoque pedagógico que consiste en emplear el tiempo fuera del aula en realizar determinados procesos de aprendizaje que tradicionalmente se hacen dentro de la misma y, por su parte, dentro del aula, con la presencia, guía y experiencia del docente, el tiempo se emplea en potenciar y facilitar otros procesos de adquisición y práctica de conocimientos, en aplicar conceptos y participar de forma creativa en los contenidos" ${ }^{\prime}$.

Teniendo en cuenta estas definiciones, podría considerarse que la clase invertida viene utilizándose por algunos docentes de forma recurrente desde hace décadas. Sin embargo, la clase invertida no consiste solo en realizar en casa lo que antes se hacía en clase, sino que supone una nueva forma de enseñanza, pues el profesor/a también puede, e incluso debe, participar en esas actividades fuera de clase. Y esto es posible a través de los medios tecnológicos, que han comenzado a utilizarse de forma habitual en la docencia universitaria en los últimos años.

La clase invertida se sustenta en los llamados escenarios virtuales de aprendizaje. Estos escenarios no solo son un artilugio para presentar información de forma virtual, sino que son un verdadero instrumento de mediación que propone una estructura de acción específica desde donde profesorado y alumnado lleven a cabo estrategias para el aprendizaje tecnológicamente mediado ${ }^{9}$.

En el contexto universitario el cambio progresivo, desde el modelo clásico de enseñanza hacia un modelo de aprendizaje activo, autónomo, al tiempo que crítico y reflexivo del alumno/a, requiere un cambio profundo de la práctica docente, en el desarrollo de la relación enseñanza-aprendizaje ${ }^{10}$. Por todo lo que estos elementos aportan, la incursión y el empleo de las TICS se antojan como pilares elementales de este tránsito. La institución educativa y el profesor/a dejan de ser fuentes de todo conocimiento, y el profesor/a debe pasar a actuar como guía de los alumnos/as, facilitándoles el uso de los recursos y las herramientas que necesitan para explorar y elaborar nuevos conocimientos y destrezas. Como resultado, el profesor/a acusa implicaciones en su preparación profesional, pues se le requiere, en su proceso de formación -inicial o de reciclaje-, ser usuario aventajado de recursos de información.

En el entorno universitario, este particular pasa a ser imprescindible, como indican FELIZ-MURIAS y RICOY "Es interesante que nos planteemos, en la educación superior, combinar diferentes estrategias metodológicas tanto por su aporte complementario como por la mejor adecuación de unas u otras en diferentes situaciones" $"$. Estos escenarios virtuales dan lugar a todo un nuevo panorama educativo

\footnotetext{
8 SÁNCHEZ RODRÍGUEZ, J.; RUIZ-PALMERO, J. y SÁNCHEZ-VEGA, E. (2017): "Flipped classroom. Claves para su puesta en práctica", Revista de Educación Mediática y TIC, Nº 6, pág. 343.

${ }^{9}$ OSUNA ACEDO, S.(2014): Escenarios virtuales educomunicativos, Icaria.

${ }^{10}$ FERNÁNDEZ MUÑOZ, R. (2014): "Entornos virtuales de enseñanza-aprendizaje para la docencia en la Universidad de Castilla-La Mancha”, Revista de Unidades de Información, Núm. 6, pág. 7.

11 FELIZ-MURIAS, T. y RICOY, C. (2008): "El desafío tecnológico en el proceso de aprendizaje universitario: los foros formativos", Revista Latinoamericana de Tecnología Educativa, Vol. 7, Núm. 1,
} 
e innovador enfoque pedagógico al que la Universidad no puede permanecer ajena, máxime si se persigue la reinvención continua y permanente de la enseñanza.

En un mundo donde el conocimiento fluye de forma inminente y multidireccional a través de las nuevas tecnologías, en un país donde los jóvenes de entre 16 y 24 años pasan 4 horas de media al día consultando sus dispositivos tecnológicos ... ¿Se puede entender la educación en esas edades sin TICs? ¿Acaso no tendrían que ser ya herramientas inherentes a la docencia universitaria? No resulta adecuado en la actualidad contemplar una educación segregada de las nuevas tecnologías.

La clase invertida tendrá un verdadero efecto motivador en el alumnado si el trabajo autónomo e incluso el trabajo en clase se realiza en entornos virtuales. Entre los mismos, pueden destacarse diferentes herramientas, tanto sincrónicas como asincrónicas: plataformas de e-learning, wikis, blogs, foros, video-conferencias, correo electrónico, instrumentos de evaluación como Kahoot o Mentimeter...

\section{Herramientas para la realización de la clase invertida.}

- $\quad$ Plataformas de e-learning

Los Learning Management System son programas que, al ser instalados en un servidor, ayudan al docente a administrar la enseñanza virtual desde cualquier ordenador conectado a Internet en todos sus aspectos: gestionar la participación de individuos, recursos, actividades, módulos, permisos, generar informes, evaluaciones, videoconferencias... etc. Este tipo de plataformas, nos remitamos a entornos gratuitos (como Moddle o Dokeos entre otros) o de pago (E-ducativa, Blacboard...), deben ser instaladas en un servidor.

\section{- $\quad$ Blogs}

Los blogs forman parte de nuestra cotidianeidad . Cada vez son más los que se apuntan a servirse de su propio espacio para compartir, promocionarse y, por qué no, vivir de ellos.

Así, podemos definir el blog es un sitio Web donde se recopilan mensajes de uno o varios autores sobre una temática en particular. Su acceso y creación es libre para todo usuario, y las plataformas de mayor presencia son Wordpress y Blogger. Como indica TORRES RIOS, las ventajas de su utilización en el aula son múltiples ${ }^{12}$ :

1. Fomenta una actitud crítica ante lo que se lee.

2. El blog pone fin al uso pasivo de Internet y aporta interactividad plena.

3. El blog proporciona a los alumnos/as una audiencia real. Normalmente los alumnos/as escriben y hacen los ejercicios para la clase y para el profesor/a, que se convierte en la única persona que lee los escritos. Sin embargo, en los blogs, los lectores/as son los compañeros/as, los cuales están interesados en comunicar algo.

\footnotetext{
pág. 58.

12 TORRES RIOS, L.(2007): "El uso de los blogs en la enseñanza-aprendizaje de E/LE”, Foro de Profesores de E/LE, $\mathrm{N}^{\circ} 3$, págs. 243-246.
} 
4. El blog puede servir de ayuda a los alumnos/as más tímidos o a aquellos que necesitan más tiempo para responder y que tienen más dificultad a la hora de participar en clase, etc.

\section{- Wikis.}

Las wikis se presentan como herramientas colaborativas que permiten elaborar de forma compartida conocimiento e información caracterizados por la apertura que presentan. Una wiki es una página web que se editar con la participación de varios usuarios, lo cual constituye su nota esencial.

Los wikis pueden utilizarse en educación como herramientas de:

1. Recopilación de información.

2. Entrega de asignaciones por parte de los estudiantes.

3. Escritura colaborativa.

4. Resolución de problemas.

5. Foros de discusión.

6. Bibliotecas

7. Práctica de habilidades de colaboración.

\section{- $\quad$ Foro}

El foro es una de las herramientas de comunicación en la que todos los miembros de la comunidad pueden aportar desde su propia perspectiva, dando lugar así al conectivismo. Es un espacio de intercambio en el que el un grupo de personas se encuentran, se expresan, interactúan e intercambian.

\section{- Correo electrónico.}

El correo electrónico es un servicio de red que permite a los usuarios enviar y recibir mensajes mediante redes de comunicación electrónica. La característica fundamental del correo electrónico es la interactividad. Se caracteriza por su inmediatez, asincronía y los mínimos conocimientos tecnológicos que se requieren para su uso.

\section{- Vídeo-conferencias}

La videoconferencia es un servicio de comunicación bidireccional y sincrónico que utiliza los nuevos canales de comunicación para propiciar un intercambio de información visual y auditiva entre dos o más interlocutores distantes. Una de sus ventajas, es la posibilidad de desarrollar estrategias colaborativas de enseñanzaaprendizaje con ellas, si bien contempla igualmente deficiencias como la posibilidad de reproducir a través de ella modelos de enseñanza tradicionales.

Todas estas herramientas pueden resultar idóneas para conseguir un trabajo autónomo por parte del alumno/a, sin embargo, en este contexto de hipercomunicación, inmediatez e instantaneidad comunicativa, los alumnos/as pueden sumergirse en la red con facilidad pero presentan carencias como, por ejemplo, la capacidad de reflexionar de 
forma crítica sobre la información que en ella encuentran. Por eso es imprescindible que adquieran una serie de competencias en este nuevo marco, entre otras ${ }^{13}$ :

- Capacidad para dar sentido a la gran cantidad de información.

- Capacidad para realizar búsquedas de información.

- Capacidad para leer y crear contenidos online.

- Capacidad para evaluar la fiabilidad de la información.

- Capacidad para detectar intereses ocultos en la información.

- Capacidad para participar en las redes y comunidades virtuales.

Como puede observarse, las herramientas para la realización de la clase invertida pueden ser múltiples, precisando algunas de ellas una mayor preparación por parte del profesor/a y otras menos. Dentro de las diferentes técnicas que he podido aplicar en los cursos académicos más recientes, las que mejor acogida y mayor motivación han provocado a los alumnos/as han sido las siguientes:

- Visionado de vídeos sobre la temática a tratar

El alumno/a visiona un vídeo que bien puede ser una clase teórica grabada por el profesor (de no más de 15-20 minutos) o un vídeo que no consista en una explicación del temario pero que les lleve a la búsqueda de información y respuestas sobre esa temática.

- Investigación sobre noticias/jurisprudencia

En este caso, la actividad anterior a la clase consiste en la búsqueda de noticias o sentencias relacionadas con la temática para una posterior discusión en la clase presencial coordinada por el docente. Resulta interesante la división por grupos de los discentes. Cada grupo debe defender una argumentación, con lo que adquieren confianza y practican la oratoria. Este instrumento es idóneo para la adquisición de conocimientos en materia de Procedimientos Tributarios.

- Realización de tareas a través del Campus Virtual

El alumno/a realiza una tarea propuesta por el profesor, que puede consistir, por ejemplo, en un comentario de jurisprudencia y son los propios compañeros/as los que, después de su exposición por parte del alumno/a en clase, revisan esa tarea y la comentan por grupos. Su realización y posterior exposición es una herramienta idónea en tanto que posibilita ejercitar y adquirir competencias tales como la capacidad de búsqueda de materiales, la síntesis, la redacción de documentos, el razonamiento crítico con aplicación de conocimientos previos.

- Utilización de aplicaciones informáticas como Kahoot o Mentimeter

Este tipo de herramientas, muy unidas a la gamificación, permiten realizar una pequeña evaluación en cada clase, no siendo para los alumnos una suerte de castigo sino más bien todo lo contrario, ya que lo toman como una especie de concurso. Kahoot es una aplicación que combina el juego y las nuevas tecnologías, que como se ha indicado son utilizadas por los alumnos/as permanentemente, pero a través de la cual se produce y se

\footnotetext{
${ }^{13}$ CANTILlO VALERO, C. (2014): "Educación y cultura digital” en OSUNA ACEDO, S. (Coord), Escenarios virtuales educomunicativos, Icaira.
} 
evalúa el aprendizaje. El profesor/a genera con esta herramienta encuestas, test y debates, que se visionan en la clase y a los que el alumnado responde a través de su teléfono móvil con una clave. La motivación de los alumnos es, según mi experiencia, mucho mayor que con cualquier otro sistema de evaluación.

Además, hemos de ser conscientes de que la simple presencia de herramientas tecnológicas no garantiza la existencia de interacción ni tampoco implica una participación activa de los individuos. Por ello, lo relevante no es su existencia o utilización en el proceso de enseñanza-aprendizaje sino la forma de uso y la participación y actitud de los sujetos implicados.

En consecuencia, si los entornos virtuales suponen un aliciente educativo para el alumnado, el docente debería saber y/o aprender a gestionar este tipo de herramientas para dar respuesta consonante y de calidad. Las herramientas a nuestro alcance pueden ser idóneas, pero deben conocerse y adaptarse a las necesidades y características de cada asignatura. Es evidente que no es lo mismo una asignatura del Grado en Ingeniería de Sistemas de Telecomunicación que del Grado en Derecho, e incluso, dentro de una misma titulación, la utilización de entornos virtuales y de la clase invertida, puede y debe ser diferente en función de las necesidades de cada área o asignatura.

\section{La conveniente utilización de la clase invertida en las asignaturas de Derecho Financiero y Tributario.}

En los planes de estudios adaptados al Plan Bolonia se diferencia entre créditos teóricos y prácticos. No dudamos de la utilidad que puede suponer esta división para ciertas áreas de conocimiento, pero en lo que respecta a la nuestra, existe una indisoluble unidad entre contenidos teóricos y prácticos, que convierten aquella separación en artificial. El Derecho Financiero no puede explicarse sin resolver un caso práctico o hacer referencia a un ejemplo o explicar la última consulta que nos ha realizado un profesional del Derecho sobre un tema complejo que tiene que resolver.

Centrándonos en la metodología específica para la enseñanza-aprendizaje de una disciplina jurídica, la formación en este ámbito supone la aprehensión de un conjunto de conceptos a través de los cuales se llegue a un conocimiento lo más completo y exacto, de tal modo que, complementada con la información, se pueda interpretar y aplicar correctamente el Derecho, se pueda ser autocrítico, valorar la legalidad existente y poder coadyuvar, en su caso, a su modificación. No se trataría de informar de manera aséptica y objetiva sobre el ordenamiento jurídico sino de analizar, más allá del Derecho positivo, la realidad existente.

La tarea formadora posee un sentido radicalmente distinto al de "transmisión de datos". Ciertamente que el alumno obtenga un grado suficiente de conocimientos e información normativa, es uno de los pilares básicos sobre los que se sustenta la docencia. Sin embargo, este conocimiento no es suficiente para la formación. El nivel de información normativa y de aportaciones de los investigadores tendrá por finalidad familiarizar al alumno con los mecanismos de comprensión crítica e integral de la realidad acotada para su estudio. La utilización de los diversos instrumentos docentes debe estar dirigida, 
básicamente, a despertar en el alumno la comprensión crítica de las conexiones de la regulación del Derecho con la realidad humana.

Una enseñanza que pretende ser más formativa que informativa, y más crítica que dogmática, debe determinar que el profesor se esfuerce en encontrar, en cada caso, el instrumento metodológico más idóneo para conseguir ese objetivo.

Entre los mismos, la clase invertida se postula, en mi opinión, como herramienta imprescindible en la enseñanza del Derecho Financiero y Tributario, tanto para la parte general como para la parte especial (derecho Financiero I y II). Para poder llegar de verdad al alumnado es necesaria la innovación metodológica, que debe pasar, irrefutablemente, por el uso de las nuevas tecnologías.

Nuestros alumnos/as pertenecen a la generación NET, generación que ha nacido desenvolviéndose con un ordenador y, entre otros rasgos, que están dirigiendo toda una revolución digital ${ }^{14}$. Se caracterizan por un alto grado de colaboración y su necesidad de controlar las máquinas. Vemos ya como no solo debemos cuestionar la tarea docente de manera cercana o alejadas de las nuevas tecnologías sino que, además, estos jóvenes parecen demandar un empoderamiento, un protagonismo, al sugerir que deben ser ellos sujetos activos en su propio desarrollo. Y es que la generación NET no se conforma con ser meros receptores de información, para ellos los medios deberían hacer lo que el usuario pida que haga permitiendo asimismo el diálogo con otras personas ${ }^{15}$.

El aprendizaje precisa por tanto de protagonismo discente, multidireccionalidad comunicativa y, por ende, de un uso adecuado de las nuevas tecnologías que favorezcan el flujo de interacción al efecto. $\mathrm{Y}$ es en este punto donde comprobamos que el ciberespacio se postula como el escenario perfecto para ello, pues es un escenario que va a hacerse realidad gracias a la llamada Web 2.0. Ello facilita el cambio de paradigma educativo en tanto en cuanto aceptemos que, efectivamente, todo usuario/a se erige como colaborador en la producción de contenidos de forma que se rompa la unidireccionalidad comunicativa docente-alumno/a.

Estas afirmaciones son plenamente aplicables a la enseñanza del Derecho Financiero y Tributario. La utilización de nuevas herramientas, y en concreto de la clase invertida, puede suponer un mejor aprendizaje del alumno ${ }^{16}$. Tradicionalmente se ha venido empleando una metodología consistente en la impartición de clases teóricas y, posteriormente, de algunas clases prácticas. Esto ha supuesto, sobre todo desde el paso de una asignatura anual a una cuatrimestral que, en muchas ocasiones, sea prácticamente imposible terminar el temario por falta de tiempo.

\footnotetext{
${ }^{14}$ BONG SEO, Y. (2002): "Dos frutos de la era cibernética: la Generación NET y los Hackers", Sincronía, revista de ciencias sociales y humanidades, Universidad de Guadalajara.

${ }^{15}$ BERNAL, C. Y BARBAS, A. (2010): "Una generación de usuarios de medios digitales", en APARICI, R.(Coord.): Conectados en el ciberespacio, UNED, págs.107-132.

${ }^{16}$ Los fallos del modelo expositivo comenzaron a ponerse de manifiesto por GIBBS en la década de los 80, sin embargo, hoy día, se sigue utilizando con asiduidad. Vid. GIBBS, G. (1981):Teaching students to learn. Milton Keynes: Open University Press.
} 
En mi opinión, esto debe modificarse. Estoy de acuerdo con MARQUÉS en que el conocimiento no se puede transmitir solo de forma verbal y que "para enseñar, lo que se debe hacer es facilitar las condiciones necesarias para que los estudiantes aprendan por sí mismos, bien mediante el descubrimiento o bien ayudándoles a pensar" ${ }^{17}$. Señalan TORTOSA YBÁÑEZ, GRAU COMPANY y ÁLVAREZ TERUEL que "la idea básica inherente a este modelo educativo sería la de promover que el alumno trabaje por sí mismo y fuera del aula los conceptos teóricos a través de diversas herramientas que el docente pone a su alcance, principalmente vídeos o podcasts grabados por su profesor o por otras personas (pero no exclusivamente), y el tiempo de clase se aproveche para resolver dudas relacionadas con el material proporcionado, realizar prácticas y abrir foros de discusión sobre cuestiones controvertidas". Sin embargo, creo que la clase invertida puede plantearse también con base en otro tipo de herramientas, y no solo en el visionado de vídeos por los alumnos, si bien este es el instrumento más utilizado y que dio origen a flipped classroom. En mi caso, son diversas las herramientas utilizadas, pues además de los vídeos, los escenarios virtuales ofrecen múltiples posibilidades.

La clase invertida es idónea para las asignaturas de Derecho Financiero, pues la parte más teórica podrían aprenderla fuera del aula sin ningún problema y dedicar después las horas de clase a la resolución de casos prácticos basados en la realidad actual, en los asuntos que verdaderamente llegan a los tribunales.

\section{A modo de reflexión.}

Tomando en consideración la virtualidad práctica de la clase invertida, puede llegarse a la conclusión de que la misma es un instrumento adecuado y útil para la enseñanza del Derecho Financiero. Sus ventajas son múltiples, ya no solo por los conocimientos adquiridos por los alumnos, sino por la obtención de múltiples competencias, como la oratoria, capacidad de defensa de una argumentación o mayor motivación.

También supone ventajas para el profesorado. Así, la evaluación en las clases invertidas es, como recuerdan SÁNCHEZ RODRÍGUEZ, RUIZ-PALMERO y SÁNCHEZ-VEGA "más objetiva y precisa puesto que el profesor tiene más datos para calificar a losalumnos debido a que ha dispuesto de más tiempo en clase para estar con ellos y comprobar sus capacidades, sus competencias" ${ }^{\prime 18}$.

Otra ventaja del uso de este sistema es la adquisición de confianza por parte del alumno/a, que se considera suficientemente autónomo y responsable como para ser activo en su proceso de aprendizaje, lo que sin duda redunda en un mejor aprendizaje por su parte ${ }^{19}$.

Se incluye también aquí la idea del aprendizaje cooperativo, que coadyuva a que todos los estudiantes asimilen el temario. Las sesiones prácticas presenciales de casos reales

\footnotetext{
${ }^{17}$ MARQUÉS, M.(2016): "Qué hay detrás de la clase al revés”, ReVisión, Vol. 9, Núm. 3.

${ }^{18}$ SÁNCHEZ RODRÍGUEZ, J.; RUIZ-PALMERO, J. y SÁNCHEZ-VEGA, E.: "Flipped classroom...", op.cit.pág. 345 .

${ }^{19}$ Sobre este particular, BURGUEÑO, J. (2016): “Una chispa pedagógica”, Padres y Maestros, Núm. 368; TOURON, J., SANTIAGO, R., DIEZ, A. (2014): Flipped Classroom. Cómo convertir la escuela en un espacio de aprendizaje, Editorial Digital Text.
} 
pueden basarse en este tipo de aprendizaje, en el que cada uno de los estudiantes puede desempeñar una función o rol diferente.

Como se ha señalado, pueden aprender los aspectos teóricos y legislativos antes de ir a clase y después realizar un verdadero proceso judicial, en el que tengan el papel de contribuyente u obligado tributario, otro de juez, otro de abogado...etc. Creo que esta metodología es, sin duda, beneficiosa para la marcha de la clase, ya que como señala DOMINGO "Se sabe que promover las discusiones en clase es una de las estrategias más comunes de aprendizaje activo. Cuando los objetivos del curso son retener la información a largo plazo, antes que utilizar cualquier fórmula expositiva se debería utilizar cualquiera otra que posibilitase la discusión entre los estudiantes" ${ }^{20}$.

Por todo lo indicado, creo que la metodología de las clases invertidas resulta idónea para las asignaturas del área de Derecho Financiero y Tributario, que tienen una gran virtualidad práctica, por lo que el énfasis debe ponerse en este aspecto por encima de las explicaciones teóricas. Si las explicaciones llevan a la rutina, a la pasividad de los alumnos, a una excesiva abstracción o a la simple repetición del manual, seguramente el aprendizaje será más reducido. En algunos casos podemos perder la atención de los estudiantes si se dedica la clase presencial a la lectura o simple exposición de la Ley Tributaria, cuando puede emplearse la misma en enseñar y en aprender cómo ha de utilizarse, y cuáles son las diferentes interpretaciones que se pueden hacer de la misma con base en casos concretos y reales. Este último objetivo puede lograrse a través de la clase invertida.

\section{Bibliografía.}

ALCUBILlA, E.A. (2016): Código de Universidades. Códigos electrónicos. Agencia Estatal Boletín Oficial del Estado.

BERNAL, C. Y BARBAS, A. (2010): "Una generación de usuarios de medios digitales", en APARICI, R.(Coord.): Conectados en el ciberespacio, UNED.

BONG SEO, Y. (2002): "Dos frutos de la era cibernética: la Generación NET y los Hackers", Sincronía, revista de ciencias sociales y humanidades, Universidad de Guadalajara.

BUNCE, D. M., FLENS, E. A., y NEILES, K. Y. (2010): "How long can students pay attention in class? A study of student attention decline using clickers". Journal of Chemical Education, 87(12).

BURGUEÑO, J. (2014): “Una chispa pedagógica”, Padres y Maestros, Núm. 368, 2016; TOURON, J., SANTIAGO, R., DIEZ, A.:Flipped Classroom. Cómo convertir la escuela en un espacio de aprendizaje, Editorial Digital Text.

CANTILLO VALERO, C. (2014): "Educación y cultura digital" en OSUNA ACEDO, S. (Coord), Escenarios virtuales educomunicativos, Icaira.

\footnotetext{
${ }^{20}$ DOMINGO, J. (2008): “El aprendizaje cooperativo”, Cuadernos de Trabajo Social, Vol. 21, pág. 234.
} 
DOMINGO, J.: (2008) "El aprendizaje cooperativo", Cuadernos de Trabajo Social, Vol. 21.

FELIZ-MURIAS, T. y RICOY, C. (2008): “El desafío tecnológico en el proceso de aprendizaje universitario: los foros formativos", Revista Latinoamericana de Tecnología Educativa, Vol. 7, Núm. 1.

FERNÁNDEZ MUÑOZ, R. (2014): "Entornos virtuales de enseñanza-aprendizaje para la docencia en la Universidad de Castilla-La Mancha", Revista de Unidades de Información, Núm. 6.

GIBBS, G. (1981):Teaching students to learn, Milton Keynes: Open University Press.

IMBERNÓN, F. (2009): "Mejorar la enseñanza y el aprendizaje en la Universidad", Cuadernos de Docencia Universitaria, $\mathrm{N}^{\mathrm{o}} 14$.

MARQUÉS, M. (2016): “Qué hay detrás de la clase al revés”, ReVisión, Vol. 9, Núm. 3 .

OSUNA ACEDO, S. (2014): Escenarios virtuales educomunicativos, Icaria.

SÁNCHEZ RODRÍGUEZ, J.; RUIZ-PALMERO, J. y SÁNCHEZ-VEGA, E. (2017): "Flipped classroom. Claves para su puesta en práctica", Revista de Educación Mediática y TIC, $\mathrm{N}^{\mathrm{o}} 6$.

SÁNCHEZ VERA, M.M.; SOLANO FERNÁNDEZ, I.M. y GONZÁLEZ CALATAYUD, V. (2016): "FLIPPEDTIC: Una experiencia de Flipped Classroom con alumnos de Magisterio", Revista Latinoamericana de Tecnología Educativa, Vol 15 (3).

TORRES RIOS, L. (2007): "El uso de los blogs en la enseñanza-aprendizaje de E/LE", Foro de Profesores de E/LE, $\mathrm{N}^{\circ} 3$. 